\title{
KRAS NM_004985.3:c.37_39delGGCinsCGT
}

National Cancer Institute

\section{Source}

National Cancer Institute. KRAS NM 004985.3:C.37 39delGGCinsCGT. NCI Thesaurus.

Code C98416.

A complex substitution where the nucleotide sequence at positions 37 through 39 of the coding sequence of the KRAS gene has changed from guanine-guanine-cytosine to cytosine-guanine-thymine 